Fecha de recepción: abril 2019 Fecha de aceptación: octubre 2019 Versión final: marzo 2020

\section{Diseño de indumentaria: Nuevas estrategias de enseñanza y modelos de innovación en el marco del consumo de moda}

Verónica Fiorini *

\begin{abstract}
Resumen: Este artículo se propone abordar ciertos cambios en los modelos metodológicos que se desarrollan en el campo de la enseñanza del proyecto en indumentaria. El impacto de las transformaciones socioculturales en los modelos de consumo, la emergencia de otras lógicas de producción de los mensajes y discursos comunicacionales en el campo de la moda son aspectos que obligan a revisar las concepciones disciplinares en la educación en diseño y en el campo profesional. Hay dos aspectos claves que se analizarán en este trabajo: los modos de pensar y enseñar el diseño y sus objetos resultantes en su relación con las experiencias de consumo que proponen en tanto valor-signo (Baudrillard). El contexto contemporáneo, incierto y fluctuante también traza un panorama de alta complejidad. La emergencia de las identidades locales, en un mundo altamente globalizado, merece ser revisado, en tanto redefine la lógica del campo disciplinar (Bourdieu) y los discursos que son legitimados en el mismo (Verón).
\end{abstract}

Palabras clave: metodologías - proyecto - diseño de indumentaria - modelos y experiencias de consumo - educación - comunicación - signo - discursividad - interdiscursividad.

[Resúmenes en inglés y portugués en la página 60]

${ }^{(*)}$ Magister en Diseño Comunicacional (DICOM) de la FADU UBA. Diseñadora de Indumentaria (UBA). Especialista en Gestión Estratégica de Diseño - Gerenciamiento de Proyecto y de Diseño Comunicacional. Magister en curso de la maestría DICOM (UBA). Profesora de la Universidad de Palermo en el Área de Moda y Tendencia en la Facultad de Diseño y Comunicación. Actualmente trabaja como diseñadora en su propia marca. Tiene varias publicaciones: "Del diseño al producto", capítulo del libro Desing de Moda, diversos olhares; "Diseño de indumentaria, aspectos conceptuales y metodológicos. Armado de colección" en Cuadernos de Cátedra FADU-UBA 2009; "Acerca del Método" en Revista Contextos de la UBA; entre otros.

Ver desfiles de la Cátedra de Verónica Fiorini en Moda en Palermo.

Pertenece a la Facultad de Diseño y Comunicación desde el año 2011. 


\section{Introducción}

Este trabajo tiene como objetivo abordar ciertos cambios en los modelos metodológicos del diseño de indumentaria en relación a las transformaciones socioculturales de consumo. También se revisarán algunas nociones ligadas a la emergencia de otras lógicas de producción y distribución de los significados de los objetos de diseño en el campo de la moda. Estos lineamientos suponen una revisión obligada de ciertas concepciones disciplinares que podemos resumir en dos grandes ejes:

1. Ciertos modos de pensar el diseño de indumentaria en sus visiones metodológicas y con respecto a la innovación.

2. Sus implicancias discursivas en relación a las experiencias de consumo que proponen dichos objetos de diseño: interdiscursividad en el diseño de indumentaria.

\section{Revisiones metodológicas en el campo del diseño de indumentaria}

Podemos afirmar que el contexto cultural y social contemporáneo, asume algunos rasgos globales y locales de cambios profundos, signados por una fuerte incertidumbre, grandes fluctuaciones y una tensión permanente entre las tradiciones disciplinares, los discursos legitimados por las mismas, y sus potenciales rupturas.

El diseño definitivamente ya no está únicamente ligado a la producción de objetos, tampoco de búsquedas puramente formales, sino a un estadío en donde lo que importa es principalmente el proceso de construcción del discurso del diseño y los significados que evocan estos objetos vestimentarios.

En este sentido, retomamos la concepción planteada por E. Verón (2004): "La noción de discurso designa, no únicamente la materia lingüística, sino todo conjunto significante considerado como tal (es decir como lugar investido de sentido)" (2004, p. 48). En este marco teórico, hay que subrayar la importancia que le otorga a las redes interdiscursivas que permiten reconocer las condiciones de producción y reconocimiento de los discursos. Es decir: "Todo discurso constituye un fenómeno de reconocimiento de los discursos que forman parte de sus condiciones de producción" (2004, p. 54).

Esta teoría retoma aspectos desarrollados por Peirce, para quien la semiosis es ternaria, social, infinita e histórica (2004, p. 56). Esta definición de discurso de Verón nos permite situar a la disciplina del diseño de indumentaria como un campo (Bourdieu) generador de discursos.

Para L. Arfuch (1997) se presenta el diseño como "trama cultural", es decir excede la actividad proyectual individual y se impone como hecho significativo (1997, p. 224). La autora señala que el diseño aparece allí donde todo está dicho, en un espesor hecho de historia, tradiciones, usos culturales, huellas, palabras de otros.

En este sentido, la metodología clásica proyectual, presenta aspectos que merecen ser discutidos y revisados a la luz de nuevas teorías, o al menos de aquellas aproximaciones que se presentan como nuevos discursos sobre como diseñar. 
En los procesos metodológicos tradicionales, herederos del discurso del diseño moderno, las fases clásicas se dividían, a grandes rasgos, en tres etapas diferenciadas:

- Fase investigativa y de detección de problemas de diseño

- Fase inventiva o creativa

- Fase ejecutiva y de concreción/verificación

Autores de distinta procedencia rescatan aspectos centrales de estos métodos proyectuales herederos del pensar de la modernidad a los largo de varias décadas del siglo XX: Maldonado, Bonsiepe, Munari, Bürdek y otros defensores de esos discursos. Incluso autores como Koberg que trabajan las nociones de circularidad y retroacción en los procesos de diseño no dejan de plantear la existencia de estas fases, aunque subviertan la lógica lineal y adviertan su complejidad creciente.

En todos estos esquemas que derivan de la concepción moderna, se advierte como fuerte influencia de la gramática de producción, el discurso funcionalista. El objeto funcional cobra valor como objeto signo, tal como lo advierte Baudrillard (1974) plantea una suerte de fachada, que vuelve opacas, al naturalizarlas, las ideas centrales del funcionalismo moderno.

En el contexto contemporáneo posmoderno, los discursos sobre las metodologías se subvierten. Lo que Baudrillard (1974) menciona como objeto valor-signo se vuelve primordial. La significación del objeto pasa a primer plano en los modelos de consumo vigentes. Ante tanta profusión de objetos y aceleración del consumo a nivel global, se advierten de este modo una serie de interrogantes:

¿De qué modo se plantean los discursos de diseño de indumentaria dentro de este modelo de obsolescencia programada acelerada que nos propone el sistema de la moda?

¿Cómo se implementan los modelos actuales de innovación en relación al pensamiento de diseño y en relación a los objetos y su producción?

¿Qué experiencias y otros discursos evocan, como se relacionan interdicursivamente con otras áreas del conocimiento y la cultura?

$\mathrm{Al}$ ahondar en los factores de innovación, autores como R. Leiro (2006), subrayan la importancia de no diseñar sobre lo ya diseñado, de proponer una ruptura innovadora a partir de la re conceptualización radical del objeto de diseño.

Este autor destaca tres fases remarcables:

1. Adaptación del diseño a los modelos culturales vigentes

2. Cambio sustancial de los significados

3. Creación de nuevos significados

Es decir, que la innovación puede presentar diferentes niveles de intensidad respecto al plano simbólico en relación al contexto sociocultural en el que está inserto.

Para Jaakko Tammela (2016), estamos en un momento de grandes transformaciones desde una perspectiva proyectual. Su planteo teórico hace hincapié en el gran cambio que significa para el diseño, tradicionalmente ligado a la lógica industrial (cuyo inicio sitúa en la Revolución Industrial). En ese contexto "la sustitución del trabajo artesanal autónomo 
por el trabajo asalariado y el uso de máquinas para la producción, aumentó drásticamente la capacidad humana" (más rápida y más fuerte como civilización). Ante la propuesta de un nuevo diseño industrial, remarca la pregunta: ¿"Estoy diseñando un nuevo vaso de agua o una manera de matar la sed?" (2016). Pensando un correlato en indumentaria uno podría preguntarse ¿Estoy diseñando un nuevo atuendo o una nueva concepción del vestir? Esta doble pregunta nos marca de manera ejemplar que la identificación del problema es siempre el pilar de la innovación proyectual. A este planteo podríamos agregar una nueva pregunta ¿Cuáles son los discursos emergentes sobre ese objeto en el plano de la experiencia y de la significación que pueden enriquecer la idea de diseño?

Para este autor diseñar es una profesión imaginativa y productiva $(2016$, p. 31) en tanto trabaja entre el mundo de las ideas y la producción de algo concreto. En la era del pensamiento digital, señala que los modelos de pensamiento se tornan inciertos, flexibles y conectados. Para Tammela es "crear lo no imaginado" pero la pregunta es ¿Cómo imaginar aquello que no fue creado hasta entonces? De qué manera los estudiantes y diseñadores pueden imaginar la innovación (2016, p. 32).

En este sentido podemos tomar la diferenciación planteada por el Profesor T. Buganza (2003) rescatada por R. Leiro (2006) quién distingue en el mundo del diseño aquellos objetos que proponen una mejora incremental y aquellos que se constituyen como innovaciones radicales. Esta definición es retomada por R. Leiro (2006, p. 207) a propósito de la marca de accesorios Swatch, quién redefine la categoría reloj como objeto de moda, principalmente.

\section{Innovación y nuevas tecnologías: interdiscursividad y diseño de indumentaria}

Las transformaciones tecnológicas alteran nuestros modos de entender el mundo, de visualizarlo y experimentarlo. El diseñador de indumentaria tiene entonces la oportunidad de repensar nuevas soluciones para nuevos problemas de diseño, y en este sentido construir nuevos discursos sobre lo que es un diseño tecnológico y también dialogar con aquellos discursos que se constituyen como "tendencias socioculturales de consumo"1. Las relaciones entre diseño y cultura son cruciales para entender cómo se producen los objetos vestimentarios y las ideas o conceptos que éstos proponen en el campo de la significación. Un buen ejemplo es la serie audiovisual Black Mirror ${ }^{2}$, en la cual se plantean distintos dilemas en una sociedad cambiante y determinada por las tecnologías en un sentido crítico. En uno de sus capítulos, las relaciones humanas hombre-mujer tienen una fecha de caducidad a partir de un dispositivo tecnológico, es decir suponen una fecha de vencimiento. En otro capítulo de la serie, una madre se obsesiona por controlar a su hija mediante un dispositivo técnico hasta anular de manera total su privacidad y su encuentro con situaciones conflictivas.

Estos discursos que parecen transitar lo ficticio y los lenguajes de la ciencia ficción son esbozados desde el campo de la indumentaria. Desarrollos de prendas que posibilitan la visión 360 grados y ejercer cierto grado de control ya constituyen espacios de exploración en el diseño de indumentaria. La inclusión de dispositivos tecnológicos en las prendas, 
que mantienen al tanto al usuario de situaciones que ocurren en sus comunicaciones ya son parte del presente. La empresa Levis (2018) ha desarrollado con éxito y con última tecnología la concreción de esta cruza interdisciplinaria.

La denominada chaqueta inteligente de Levis, posee sensores y botones que controlan el móvil (denominado Project Jacquard, con Levis commuter Trucker Jacket). El ingeniero del proyecto Iván Poupyrev plantea que dicho producto ha sido desarrollado para "ayudarte a controlar tu vida digital mientras estás en el mundo real” (Bohn, 2017). Dentro del mundo de los wearables, dicho producto, se puede lavar fácilmente. Con solo determinados gestos o hacer toques en la ropa puede suspenderse la música o recibir indicaciones de google maps, o escuchar mensajes leídos por un asistente virtual.

En Argentina, también se ha desarrollado un dispositivo en la indumentaria que permite detectar caídas en personas mayores a larga distancia, implemento que busca ayudar tecnológicamente en términos de cuidado de la salud corporal.

De este modo, el cuerpo se vuelve no solo soporte de la vestimenta, sino de los dispositivos móviles de una manera más mimética. También es posible afirmar que este tipo de prendas anticipan un cambio en la gestualidad del usuario, una innovación en los movimientos del mismo y por ende en la comunicación no verbal. Del mismo modo podemos afirmar que varias películas o series de ciencia ficción como Black Mirror forman parte de la gramática de producción de estos discursos del diseño. De eso se trata la inter-discursividad en el campo cultural.

En otro caso emblemático, las zapatillas Nike que se ajustan solas sin intervención del usuario (fantasía desplegada en la legendaria película de los años ochenta Volver al futuro) ya fueron presentadas durante 2017 por la marca. Es decir, la búsqueda de objetos que actúan sin el usuario, actúan por su cuenta, de modo independiente. Este concepto es una idea de profundo impacto que nos demuestra el lazo indisoluble entre los discursos del diseño y la cultura. Los modos en que se esbozan los imaginarios de futuro están en diálogo permanente con las imágenes que construyen los discursos actuales del diseño, demostrando plenamente la inter-discursividad en los objetos vestimentarios.

Con respecto a la innovación, en diseño de indumentaria, también son dignos de remarcar los casos en donde se presentan rupturas con los discursos imperantes del campo. En este sentido, en los procesos de diseño y de enseñanza del diseño, son muy importantes los cambios discursivos para producir un nuevo producto, un nuevo desarrollo proyectual. En el caso de las camperas de la marca Uniqlo ${ }^{3}$ se evidencia claramente como un cambio discursivo genera un cambio material y formal en las prendas.

En vez de presentar la idea de un abrigo, desde mi punto de vista, presentan la idea de la desmaterialización, es decir, "un objeto de abrigo como una vestimenta que casi no existe por ser tan liviana”. La liviandad y la compresión son la antítesis del abrigo. Por eso la resultante fue un producto con alta tasa de innovación, muy imitado por otras marcas a nivel global. Que luego fue imitado en la industria de manera exponencial.

En el caso del diseño de accesorios, el desarrollo de otro tipo de zapatillas Nike que pueden ayudar a contabilizar los pasos, a seguir el ritmo cardíaco, etc. no solo promueven un cambio tecnológico, sino que proponen un discurso de control sobre el propio cuerpo. Es decir, la indumentaria controla a su usuario y no al revés. Lo informa y pasa de ser un objeto inanimado a tener "vida propia". 
La detección y búsqueda de estas performances de estos productos están asociadas a una profunda observación de los usuarios, sus inquietudes, sus preferencias, gustos e inclinaciones. En este sentido resulta relevante el abordaje metodológico que proponen algunos autores vinculados al design thinking como E. Lupton (2011).

En algunos de los planteos esbozados por el denominado design thinking, planteados en su obra, se reflejan, de modo un tanto esquemático, aquello que el campo proyectual ya ponía en práctica: conceptualización, jerarquización, resignificación, deconstrucción de los problemas, innovación radical e incremental. Nuevas herramientas como mapas conceptuales, volcado visual de datos pero una estructura similar en términos de pensamiento proyectual. Para el Innovation Factory Institute de Barcelona, (2013), el design thinking se presenta como una aproximación metodológica para los procesos de innovación en diseño. Se centra en el proceso más que en el producto final y apunta a enfocar a las personas como eje clave de observación por parte del diseñador. La comprensión del usuario y su interacción con el producto son el punto de partida para la innovación proyectual. Pero a pesar de centrarse más en el proceso, no se aleja drásticamente de los procesos proyectuales clásicos, sino que los complejiza, los vuelve más permeables a los fenómenos sociales y culturales y menos lineales.

Si en los métodos tradicionales de diseño las etapas centrales giraban en torno al estudio de problemas de diseño y sus condicionantes, creación y resolución técnica-verificación, podemos afirmar que en los modos de diseño actual existen instancias más profundas de la relación diseño-usuario-experiencia-discurso. Por otra parte, en estos abordajes se vuelven claves los términos y las palabras identificadas y utilizadas. El trabajo sobre mapas conceptuales que orientan, a modo de guía, a través del lenguaje, una búsqueda más certera, con un fuerte lazo con las personas que utilizarán esas prendas de vestir o esos accesorios de diseño.

Para la diseñadora italiana Nanni Strada (2009) la relación diseño y lenguaje es estrecha y determinante. En sus desarrollos pedagógicos respecto al proyecto sitúa la concepción del lenguaje como un aspecto central en la creación de indumentaria con alto valor desde el punto de vista del sentido.

En este sentido, podemos avanzar pensando que discursos sociales y culturales posibilitan la lectura de que ese objeto es posible y deseable en términos conceptuales? ¿Qué experiencias evocan y cuales proponen de manera concreta? ¿Quiénes pueden estar interesados en esa gama de experiencias y como se enraizan en otras creencias, valores e ideas?

Una marca como Melissa plantea una serie de experiencias ligadas a un consumo lúdico donde el calzado es casi un juguete, un objeto artificioso, alegre, con reminiscencias infantiles.

Dentro del campo de diseño de autor ocurre algo similar. Los discursos se construyen sobre la base de deconstruir aspectos del rubro, de esa categoría de objetos. El planteo posmoderno en el diseño nacional argentino, encuentra un exponente interesante en el diseñador Marcelo Giacobbe. El concepto de un nuevo lujo artesanal, democratizado, no ostentoso y con un fino y delicado rasgo glamoroso se puede enlazar discursivamente con la idea innovadora de deconstruir la concepción tradicional de la ropa de noche, de la alta costura. Es decir, sigue siendo indumentaria a medida, con apliques, bordados, pero con una nueva aproximación discursiva. 
Para D. Norman (2005) los principales componentes del diseño de producto son: la usabilidad (o su ausencia), la estética y la utilidad práctica. "Pero lo que muchos diseñadores no perciben es que existe un fuerte componente emocional en el modo en que los productos son diseñados y utilizados". La teoría de Norman es que este factor emocional puede ser mucho más decisivo que el aspecto práctico de los objetos de diseño a la hora de elegirlos. Además de visualizar la interdiscursividad en relación a las nuevas tecnologías, también es pertinente recalcar casos de interdiscursividad en el diseño asociado a las problemáticas políticas, sociales y ambientales. Son aquellas propuestas, cuyo fin decisivo es la denuncia, la acción y también en ciertos casos la colaboración directa en problemáticas de índole social. En el campo pedagógico en Argentina, podemos citar el ejemplo de varias alumnas de la Universidad de Palermo (Diseño VI, 2017) que desarrollaron, bajo la supervisión de Fábrica (organismo dependiente de área gubernamental) y de la materia diseño VI, prendas de lluvia para personas de bajos recursos de las zonas inundables del litoral argentino. En este caso, la problemática central era relacionar una producción de una marca propia que incluyera una línea de fuerte impacto social. En términos sustentables, podemos remarcar que el diseñador-estudiante articula su producción con las necesidades de su región, de su cultura. El material de base eran banners de vinilo reciclados, y esa materia prima era procesada y repensada en clave funcional. La posibilidad de compactar el producto, que sea de fácil traslado y que pueda articularse con objetos escolares para que los estudiantes guarden, fueron algunos de los desafíos que asumieron desde el punto de vista metodológico. En términos discursivos, se buscó aunar la conciencia ambiental y social con la producción de una marca dentro del sistema de la moda. Es decir, que metodológicamente se vinculan problemas de diseño, necesidades locales sociales y ambientales.

De ese modo, se establece una formación consciente de los cambios contextuales.

En Latinoamérica, la mirada política de la moda contemporánea se presenta de un modo innegable en las colecciones de Ronaldo Fraga. Cuando muestra su colección (2016) inspirada en los discursos del amor, que el mayor acto de rebeldía en el contexto contemporáneo es justamente hablar del amor, marca un discurso potente y provocador en el campo de la moda. En términos interdiscursivos, sus anteriores colecciones tocan temas urticantes, desde el mundial de fútbol, las favelas, y problemáticas sociales y políticas.

También podemos afirmar que existe otra aproximación digna de remarcar, en cuanto a un posicionamiento que cuestiona las mismas bases del campo, es decir la obsolescencia programada de los productos vestimentarios. Este posicionamiento va en un sentido discursivo, en contra de las reglas básicas del sistema, pero tal como señala F Davis, se constituye como una "falsa" negación del consumo de moda (1992). Para ejemplificar esta postura, señala la austeridad y el minimalismo como modo de freno ante el sistema de la moda. Desde el modelo impuesto por Calvin Klein en los años 90, las propuestas de la primera etapa del diseñador Alexander Wang, o marcas argentinas como Kostume o Dubié, varias marcas desafían desde una visión estética, de economía de recursos materiales y formales, la noción tradicional de la indumentaria de moda.

$\mathrm{Al}$ respecto, resulta necesario subrayar el lazo conceptual entre el minimalismo y el funcionalismo moderno. En ambos se repite la abolición del ornamento, el énfasis en la estructura, aunque a veces se presente deconstruída y en las formas limpias. La experiencia pro- 
puesta desde este tipo de diseño es consumir menos cantidad de prendas, más duraderas, de mejor calidad y que comunicacionalmente emanen una idea de pureza y simplicidad. Desde otra óptica, en las antípodas del neo minimalismo, también se sitúa la exaltación del denominado mal gusto que juega con cierta rebeldía respecto al campo de la moda, pero dentro de su lógica. Marcas y diseñadores posmodernos se rebelan contra el gusto imperante y bucean en categorías relacionadas con el neo kitsch o el denominado gusto "vulgar", a nivel global y local. En dichas marcas abunda la cita histórica, la exageración del ornamento, y la valoración de lo artesanal en un sentido glamoroso. Marcas como Prada, Versace, $D y G$, y en una escala más masiva en Latinoamérica, la marca argentina Jazmín Chebar intentan abordar este tipo de discursos que desafían las categorías impuestas por la moda como campo.

\section{A modo de recapitulación}

Lejos de cerrar el debate, estas líneas solo se proponen subrayar algunas problemáticas desplegadas a lo largo de este texto, que constituyen un punto de partida para futuros trabajos teóricos dentro del campo.

A lo largo de este desarrollo teórico se buscó puntualizar algunos cambios en los modos de pensar el diseño de indumentaria desde una óptica metodológica, focalizando en algunas transformaciones respecto al modelo de proyecto moderno y ahondando en las experiencias actuales en el campo del diseño contemporáneo a nivel local y global. A través del estudio de casos de ambos contextos, se ha vislumbrado la creciente complejidad discursiva en el campo de la moda.

Con ese objetivo se relevaron algunos aspectos relacionados al diseño de experiencias, diseño emocional y design thinking, como algunos modelos de pensamiento que permiten vislumbrar otras aristas metodológicas dentro del área disciplinar.

Por otra parte, se intentaron dilucidar distintos enfoques sobre los modelos de innovación en el campo de la moda, abarcando cuestiones relativas a la relación diseño, discurso y lenguaje, y haciendo hincapié en la noción de interdiscursividad presente en la disciplina del diseño y en el campo de la moda. Algunos de estos diálogos interdiscursivos abarcaron relaciones entre diseño y tecnologías, así como diseño, política y sustentabilidad asociada a la identidad.

La complejidad comunicacional en el diseño de moda es exponencial, por la cantidad de información en la era digital, por la interacción de los usuarios con las marcas a través de las redes sociales, por el tráfico creciente de productos de origen mixto (diseño, producción y distribución son mixtos). En este panorama, los discursos emergentes de los diseñadores conectados con realidades locales y de pensamiento crítico respecto a las identidades regionales cobran visibilidad y relevancia (Fraga, Juana de Arco, Giacobbe). También aquellas marcas de renombre global (como Levis, Nike y Uniqlo), que dialogan con los cambios profundos que atraviesan los comportamientos de los consumidores, logran desarrollos exitosos y significativos.

En virtud de lo expuesto cobra relevancia inusitada la frase de E. Morin: "Los analfabetos del siglo XXI no serán los que no sepan leer y escribir, sino los que no puedan aprender, 
desaprender y reaprender" (2011, p. 144). La idea retomada por Verón de una semiosis infinita, mutable y abierta, donde no hay punto de llegada preciso ni cierre (de la teoría de Pierce) es ilustrativa de la compleja interdiscursividad que enfrentan los diseñadores de indumentaria. Esto significa comprender la complejidad del sentido de los objetos que diseñamos y su relación con las experiencias, emociones, ideas y discursos que la indumentaria atraviesa, tanto en su proyectualidad como en el campo profesional.

\section{Notas}

1. El término tendencia es retomado de la categoría propuesta por el sociólogo F. Morace.

2. Serie emitida por Netflix (capítulos temporada 4).

3. Esta serie de prendas ultralivianas constituye un ejemplo de un producto altamente exitoso por un cambio no sólo estético sino de sentido en el campo del diseño.

\section{Bibliografía consultada}

Arfuch, L. y otros autores. (1997). Diseño y comunicación. Teorías y enfoques críticos. Buenos Aires: Editorial Paidós, estudios de comunicación.

Baudrillard, J. (1974). Crítica de la economía política del signo. México: Siglo Veintiuno Editores.

Baudrillard, J. (1969). El sistema de los objetos. Madrid: Editorial Siglo Veintiuno.

Bonsiepe, G. (1993). Del objeto a la interfase. Mutaciones del diseño. Buenos Aires: Ediciones Infinito.

Bourdieu, P. (2011). Cuestiones de sociología. Madrid: Editorial Akal.

Bourdieu, P. (2010). El sentido social del gusto. Elementos para una sociología de la cultura. Buenos Aires: Siglo Veintiuno Editores.

Davis, F. (1992). Fashion, culture and identity. Estados Unidos: Editorial University Chicago Press.

Faerm, S., Veneziani, M., Cuervo, M. comp. Fiorini, V. y otros autores. (2015). Interpretando el pensamiento de diseño del siglo XXI. Capítulo de mi autoría: Tendencias de consumo, innovación e identidad en la moda. Ed. Cuadernos del Centro de Estudios en Diseño y Comunicación (Ensayo).Buenos Aires, editado por la UP y Parsons New School for Design.

Lupton, E. (2012). Intuición, acción, creación. Graphic Design Thinking. Barcelona: Editorial GG Diseño.

Leiro, R. (2006). Diseño, estrategia y gestión. Buenos Aires: Ediciones Infinito.

Maldonado, T. (1977). El diseño industrial reconsiderado. "El proyecto moderno". Barcelona: GG Diseño.

Norman, D. (2005). El diseño emocional. Barcelona: Ediciones Paidos.

Tammela, J. (2016). A revolucáo do Design.Conexóes para o século XXI. Brasil, editado por IED. Verón E. (2004). Fragmentos de un tejido. Barcelona: Editorial Gedisa. 


\begin{abstract}
This article aims to address certain changes in the methodological models that are developed in the field of teaching the concept of project in Fashion. The impact of sociocultural transformations in consumer models, the emergence of other logics of message production and communicational discourses in the field of fashion are aspects that force us to revise the disciplinary conceptions in education in design and in the professional field. There are two key aspects that will be analyzed in this work: the ways of thinking and teaching the design and its resulting objects in their relationship with the consumer experiences they propose as a sign-value (Baudrillard). The contemporary, uncertain and fluctuating context also traces a panorama of high complexity. The emergence of local identities, in a highly globalized world, deserves to be revised, as it redefines the logic of the disciplinary field (Bourdieu) and the discourses that are legitimized in it (Verón).
\end{abstract}

Keywords: methodologies - project - design of clothing - models and experiences of consumption - education - communication - sign - discursivity - interdiscursivity.

Resumo: Este artigo tem como objetivo abordar algumas mudanças nos modelos metodológicos cos desenvolvido no campo do ensino do projeto em roupas. O impacto das transformações socioculturais nos modelos de consumo, a emergência de outras lógicas de produção de mensagens e discursos comunicacionais no campo da moda são aspectos que nos obrigam a rever as concepções disciplinares no design e no campo profissional. Existem dois aspectos-chave que serão analisados neste trabalho: as formas de pensar e ensinar o design e seus objetos resultantes em sua relação com as experiências do consumidor que eles propõem como valor -assinar (Baudrillard). O contexto contemporâneo, incerto e flutuante também traça um panorama de alta complexidade. O surgimento de identidades locais, em um mundo altamente globalizado, merece ser revisado, pois redefine a lógica do campo disciplinar (Bourdieu) e os discursos que são legitimados no mesmo (Verón).

Palavras chave: Metodologias - projeto - design de roupas - modelos de consumo e experiências - educação - comunicação - signo - discursividade - interdiscursividade.

[Las traducciones de los abstracts fueron supervisadas por el autor de cada artículo] 